\title{
Possible COLREGs Failures under Digital Helmsman of Autonomous Ships
}

\author{
Lokukaluge P. Perera \\ UiT The Arctic University of Norway, \\ Troms $\varnothing$, Norway, \\ Prasad.Perera@uit.no.
}

\author{
Bjørn-Morten Batalden \\ UiT The Arctic University of Norway, \\ Troms $\varnothing$, Norway, \\ Bjorn.Batalden@uit.no
}

\begin{abstract}
Autonomous navigation will play an important role in the future of the shipping industry. Hence, this study illustrates several concepts that should support the navigation side in relation to the collision avoidance of autonomous ships. The concept of system intelligence, i.e., cloned by human navigators, as the digital helmsman to navigate future vessels is discussed in the first part of this study. That can provide an adequate solution to the ship controllability problem. A collision avoidance framework. i.e., based on fuzzy logic, developed to support the digital helmsman is discussed in the second part of this study. The same collision avoidance framework complements the digital helmsman. Future vessels will navigate in a mixed environment, where manned, remote controlled and autonomous vessels are interacting. Hence, the proposed collision avoidance framework, as a decision support feature based on the respective navigational rules and regulations, should support both humans as well as systems to make appropriate actions in such a navigation environment. It is expected to have an adequate consistency between human and system collision avoidance actions to preserve the integrity of system level intelligence. In the third part of this study, the consistence between human and system decisions/actions in critical collision avoidance situations with the main intention of identifying possible regulatory failure situations in a simulated environment are investigated by using the same collision avoidance framework.
\end{abstract}

Keywords-Autonomous Ship, Digital Helmsman, COLREGs, Ship Maneuvering, Collision Avoidance, Regulatory Failures, Crash Stop Maneuvers, Critical Collision Conditions.

\section{INTRODUCTION}

Autonomous ships will introduce the next technological revolution in the shipping industry as a part of Shipping 4.0 [1]. However, the required technologies and infrastructure to make autonomous navigation in deep-sea shipping are still underdeveloped. Therefore, it is expected that the firstgeneration autonomous vessels will support short-sea shipping type activities, i.e., ferries and inland shipping. Furthermore, the existing infrastructure in ports and inland waterways can also be adopted to make short-sea autonomous shipping a reality in the near future. However, close vessel encounters in congested ship routes can be one of the biggest challenges that the shipping industry can face in such situations. Hence, collision avoidance in close ship encounters should be an important research concept to study in the future, and that can improve the navigation safety of future vessels in short-sea shipping.
The outcome of a collision risk assessment in a vessel encounter situation can be used to identify possible collision or near miss situations. In general, the respective collision risk in realistic ship encounters is difficult to assess, accurately because not only vessel positions but also their orientations should also be considered into the same. Inaccuracy in vessel navigation details can degrade the collisions risk assessment process, and that may increase the risk of ship collisions. The outcome of the collision risk assessment process can eventually be used as a decision supporting feature for future ships, where intelligent systems can make navigation decisions. Such a decision support feature should be based on the respective rules and regulations of ship navigation. The International Regulations for Preventing Collisions at Sea 1972 (COLREGs) facilitates to avoid ship collisions in open sea areas. However, additional local navigational rules and regulations can be enforced on vessels especially in confined waters and maritime traffic lanes. The respective system intelligence under the decision support feature should be evaluated to verify their decision consistency and regulatory capabilities in handling various ship navigation situations, especially under mixed environmental conditions, where remote-controlled, autonomous and manned vessels are interacting. The outcome of this process can improve the navigation safety of future autonomous vessels.

\section{SHIP INTELLIGENCE}

\section{A. Mixed Environment}

The first-generation short-sea autonomous ships will navigate in a mixed-environment with close ship encounters. The vessel interactions in such environments can compromise navigation safety since both humans and systems are making the respective decisions [2]. Humans are considered as rational decision-makers, because humans systematically select possible choices based on reasons and facts. On the other hand, system-based decision-making processes that can mimic human behavior are yet to be formulated and that will be purely based on a given set of rules. However, it is expected that onboard intelligent systems can develop human friendly decision-making capabilities in the future to support autonomous vessels. Some decisions can be executed as collision avoidance actions through rudder and propeller control systems, therefore ship controllability in relation to vessel seakeeping and maneuvering behavior should also be considered. 


\section{B. Ship Controllability}

Vessels are considered to be under-actuated systems associated with heavy body inertia. In general, it is impossible to achieve the full controllability of ocean-going vessels from rudder and propeller control systems, especially under rough weather conditions. However, a limited number of overpowered vessels may be able to preserve their controllability under calm weather conditions. Such vessels are not the scope of this study. Various unexpected and undesirable motions relate to vessel seakeeping and maneuvering behavior can be expected in under-actuated vessels, i.e. due to the respective hydrodynamic and wind forces and moments on the hull and superstructure. Furthermore, vessels are slow responsive systems with considerable time-delays. Therefore, vessels can take considerably longer periods to response to rudder and propeller changes and that can create additional difficulties in ship controllability.

Even though various advanced controllers have been proposed by the research community to address such challenges in ship navigation, that has never been implemented to realistic ocean-going situations mainly due to system-model uncertainties. The outcome is that the respective controllers cannot preserve their robustness and stability in realistic oceangoing conditions. It can be concluded that ship controllability is a complex problem and lack of adequate mathematical models may result in ocean-going vessels with inadequate controllability. The same issues in ship navigation have often been ignored by the research community. Therefore, adequate solutions to overcome the challenges in under actuated ships have yet to be investigated by the future researchers.

\section{Digital Helmsman}

Machine learning and artificial intelligence-based techniques, i.e., deep neural networks, can provide a smart solution to the ship controllability problem [3]. Deep neural networks, as a part of deep learning, can provide adequate system intelligence into ocean-going vessels to navigate as agent-based systems by having a human behavior based mathematical framework. However, these deep neural networks should be trained by human navigators, similar to the autonomous car [4] to develop such behavior models. In general, deep neural networks can capture human helmsman behavior through large-scale real-world ship navigation data sets. Hence, an agent based on deep learning is classified as the digital helmsman due to their digital nature and human behavior in ship navigation. Since future vessels will be navigated by the digital helmsman, the respective collision avoidance actions should also be executed by the same system intelligence. Therefore, it would be an advantage, if the proposed decision support feature for ship collision avoidance used by human navigators, can also be used by the digital helmsman to navigate future vessels.

\section{Collision Avoidance}

Collision avoidance will be an important part of autonomous vessels, especially in close ship encounter situations. It is expected that the digital helmsman executes the respective collision avoidance actions based on the decision support feature. Ocean-going vessels are not navigating in well-defined sea routes, therefore one vessel can observe other vessels with various course-speed conditions with different headings in its vicinity. The digital helmsman should be aware of such vessel encounters and take appropriate actions to avoid a possible collision or near miss situations, especially in mixed environments. Since these vessels can have various interactions within the navigation environment, the collision avoidance actions taken by both humans as well as systems should have an acceptable level of consistency. The important concepts discussed previously in relation to collision avoidance in autonomous ships are summarized in Figure 1.

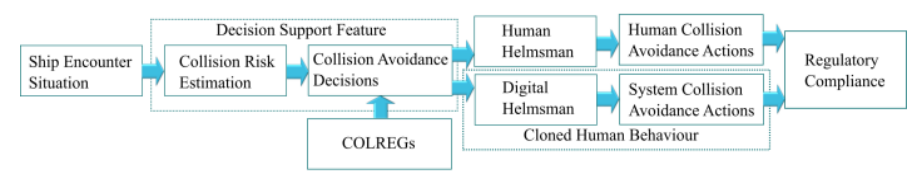

Fig. 1. The important components of a ship collision avoidance framework.

Each ship encounter situation should be analyzed to estimate the respective collision risk. Then, the same collision risk should be used to formulate appropriate collision avoidance decisions. It is expected that the respective collision avoidance decisions should also be similar to human decisions. Since the digital helmsman is trained by human navigators, the respective decisions should also be understood by such systems. The combination of those two components, i.e. the collision risk estimation and collision avoidance decisions, can be categorized the decision support feature. That can be used by both humans as well as systems to make appropriate collision avoidance actions. The collision avoidance actions that can be taken by a ship navigator divided into two categories: speed change (to increase or decrease) and course change (to the starboard and port). That should be done through ship propulsion and rudder control systems. In general, the alteration of course is the most preferred collision avoidance action, since low speed conditions can also reduce ship maneuverability.

Since both humans and systems convert these collision avoidance decisions into actions, their regulatory compliance should also be evaluated. Therefore, any inconsistency between human and system collision avoidance actions can be eliminated to preserve the integrity of system level intelligence. This study investigates the respective regulatory compliance in relation to possible COLREGs failure situations under several simulated system decision making situations in ship navigation. That outcome can also help to improve the behavior of the digital helmsman and identify possible modification on the respective navigation rules and regulations to support future vessels.

\section{THE COLREGS}

\section{A. Decision Consistency}

It is expected that the first-generation autonomous vessels should follow the existing rules and regulations of the COLREGs due to the mixed environment. However, the COLREGs will be interpreted by both humans as well as systems during these ship encounters. To avoid possible collisions or near-miss situations, the interpretations should have a high level of consistency. Developing a collision avoidance framework with adequate consistency between 
human and system interpretations can be an appropriate solution. A mathematical framework based on fuzzy logic, i.e., a computational intelligence approach, that has been developed previously to support the same consistency between human and system interpretations of the COLREGs is considered in this study [5].

\section{B. Fuzzy Logic in COLREGs}

Since fuzzy logic can imitate a human type decisionmaking process, many human friendly industrial applications are based on the same [6]. Fuzzy logic is considered as the method to formulate the COLREGs into a human friendly decision support feature. Hence, the decision support feature can support the digital helmsman to navigate future vessels and avoid a possible collision or near miss situations. The respective fuzzy membership functions are formulated in a way so that the ship navigators' understanding of the COLREGs rules and regulations in relation to various ship encounter situation can be captured into a mathematical framework. Furthermore, this mathematical framework should be exhaustive, consisting of all possible ship encounter situations, the input fuzzy membership functions, the collision avoidance decisions, and the output fuzzy membership functions - that should be taken by ship navigators.

One should note that the collision avoidance decisions should be based on the COLREGs rules and regulations. Those rules and regulations are incorporated by mapping the input and output fuzzy membership functions of a Mamdani type Fuzzy inference system [7]. The Mamdani type Fuzzy membership functions can represent an IF-THEN-ELSE type rule-based logical structure. Hence, that can provide a good mathematical framework to capture the respective regulatory structure of the COLREGs. The COLREGs should be aligned with the fuzzy membership functions to achieve these objectives, as mentioned before. Further details on this fuzzy logic based mathematical framework for ship collision avoidance are presented in the work of Perera et al. [5, 8]. The same framework can support the implementation of the COLREGs rules and regulations under the digital helmsman. While systems are making decisions, there is a possibility that some regulatory failures can occur through the same framework and that should be investigated further. Hence, the decision support feature is evaluated in a simulated environment for the regulatory failure situations, assuming that the COLREGs are interpreted by the digital helmsman.

\section{Collision Risk Estimation}

Any multiple vessel encounter situations can be separated into multiple two-vessel encounter situations. This collision avoidance framework is developed with respect to a twovessel encounter situation (see Figure 2). It is also assumed that these are power-driven vessels. The vessel that has the collision avoidance framework is named the "own vessel" and the other vessel is named the "target vessel". It is expected that both own- and target vessels are approaching a close ship encounter situation, where the respective collision risk should be estimated (COLREGS, 1972 - Rule 7 Risk of collision). The collision risk in a ship encounter situation should take account of the respective collision avoidance decisions (see Figure 1) (COLREGS, 1972 - Rule 8 Action to avoid collision) [9].

The navigation area of the own vessel is divided into three regions: general collision risk region, critical collision risk region and vessel domain. These regions are introduced to formulate the COLREGs into fuzzy logic adequately through the respective membership functions. Three simplification steps are introduced in this situation (Figure 2). One assumption is that the vessels are moving in straight-line trajectories. Secondly, the vessel course-speed vectors and headings are in the same direction [10]. Even though vessels navigation with complex maneuvering trajectories have been presented in the literature [11], such situations are considered as beyond the scope of this study. Thirdly, the study does not include situations for which Rule 19 Conduct of vessels in restricted visibility, as this rule cross out the rules 11 to 18 (Conduct of vessels in sight of one another).

One should note that having a trajectory intersection point between the own and target vessels does not mean that it is a possible collision or near-miss situation. These vessels can pass the intersection point at different time intervals with relatively safe distances. Therefore, the collision risk between these vessels can be negligible. If the collision risk is negligible, none of the vessels should make any course or speed changes. The vessel should keep their course-speed vectors in such situations concerning the COLREGs. However, this concept is often ignored by the research community, especially under path planning type algorithms, where unnecessary course speed change actions can be taken by the respective vessels.

A possible collision situation between two vessels can be determined by the relative bearing (see Figure 2), i.e., if any relative bearing changes cannot be observed within a considerable time period and the distance between the vessels diminish, the vessels are heading towards a possible collision or near miss situation. This bearing and distance concept is transformed into the relative motion between two vessels, where the relative navigation trajectory of the target vessel with respect to the own vessel is estimated. The concept is adopted to illustrate the mathematical definition of a possible ship collision situation under this framework: if the relative navigation trajectory of the target vessel intercepts the own vessel domain, then that situation is categorized as a collision or near-miss situation. In general, the relative course-speed vector of the target vessel can be used to estimate the relative navigation trajectory of the target vessel. The distance and time for the closest point of approach both in relative and actual scales in a close ship encounter situation can be calculated to quantify the respective collision risk.

\section{Head-on and Overtaking Situations}

Vessels can have various navigation limitations and that should be considered during collision avoidance maneuvers. Ocean-going vessels can have speed limitations due to their engine-power configurations. A vessel with a large displacement also have a large moment of inertia, making it 
time consuming to alter its speed. This applies for both acceleration and deceleration situations. Furthermore, vessels under slow speed conditions can lose their maneuverability. This is one of the reasons the COLREGs has highlighted safe speed to avoid collision or near-miss situations (COLREGs, 1972 - Rule 6 Safe speed). These speed conditions can also relate to vessel maneuverability with special reference to stopping distance and turning ability. Since the turning ability of a vessel relates to its speed, adequate ship speed should be maintained to preserve vessel maneuverability. One should note that the vessel with inadequate maneuverability can result in possible collision or near miss situations with COLREGs violations.

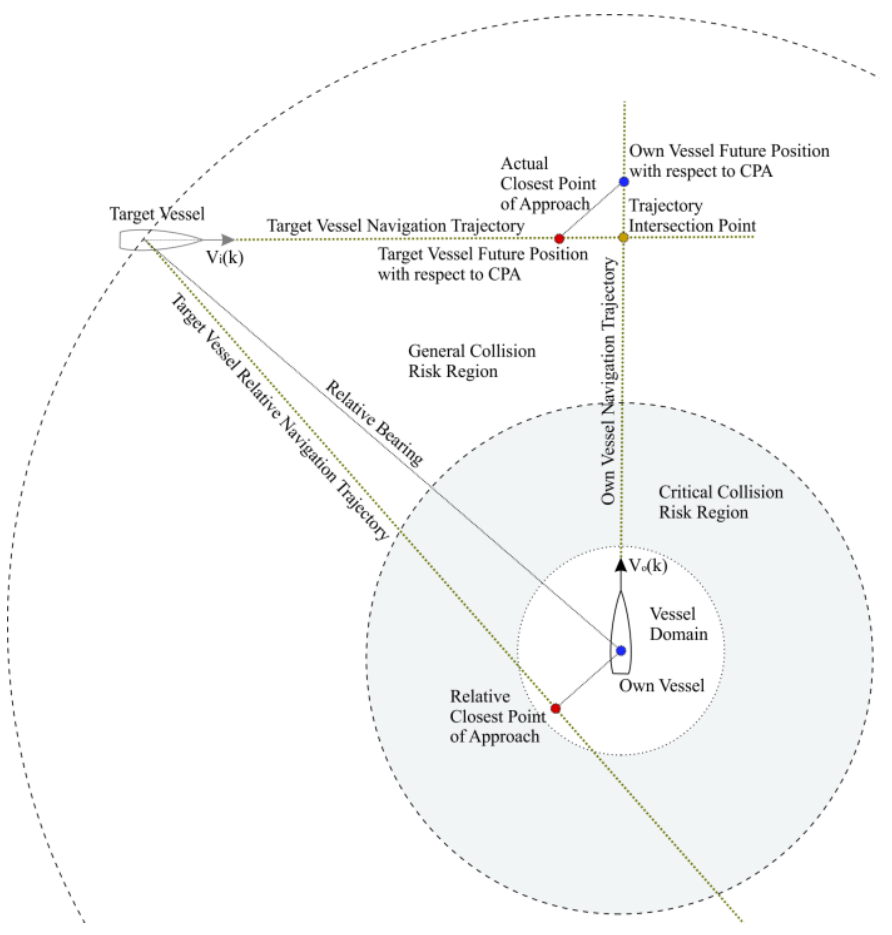

Fig. 2. Mathematical framwork for collsion risk estimation

This section discuss the respective collision avoidance actions in accordance with the COLREGs for the general collision risk region (see Figure 2). In general, three ship encounter situations are illustrated in the COLREGs: overtaking, head-on and crossing. One should note that these situations can represent all possible ship encounters.

Even though the COLREGs does not specify the respective side one vessel should overtake another (COLREGs, 1972 Rule 13 Overtaking), the outcome of this study suggests that the overtaking vessel should pass on the port side of the overtaken vessel (with enough time and distance). That outcome is influenced by the head-on situation, where the vessels should pass each other port-to-port (COLREGs, 1972 Rule 14 Head-on situation). Rule 13 differs from Rule 14 and 15 as the rule applies even when there is not a risk of collision - it is enough to be overtaking the other vessel, coming up with the vessel ahead. The nature of the fairway and navigational waters in combination with the traffic situation may make it necessary to pass on the starboard side, but in this study, the passing side for an overtaking vessel should be on the port side. Passing on the port side limits the vessel's opportunity to make alteration of course to starboard for other vessels. On the other hand, passing on starboard side limits the overtaken vessel to make course alterations to starboard. Therefore, a consistence between these two similar ship navigation situations should be established by adopting the port passing concept. There is not a definite angle defining the difference between a head-on situation and a crossing situation, and this has resulted in several collisions. Rule 14 do state that if there is doubt about the situation, it shall be reacted as if the situation is a head-on situation. The relative motion of one vessel with respect to the other vessel has considerable similarities in both situations. Therefore, an inconsistence, i.e. a possible regulatory failure situation, between these two similar ship navigation situations can avoided by the port-to-port approach, specially under the digital helmsman.

\section{E. Crossing Situations}

The most complex collision avoidance actions in ship navigation can be executed during crossing situations (COLREGs, 1972 Rule 15 Crossing situation). Such a situation with additional navigational details is presented in Figure 3 and the COLREGs provide adequate navigation guidelines to navigate in such vessel encounter situations. It is regulated that the vessels coming from the starboard have the priority to navigating in ship encounter situations. The ships have a higher and lower priority in an encounter situation are called the stand-on and give-way vessels, respectively (see Figure 2). Therefore, the give-way vessel (COLREGs, 1972 Rule 16) should take early collision avoidance actions to avoid a possible collision or near-miss situation and the stand-on vessel should keep its course and speed (COLREGs, 1972 Rule 17). A succession of small course and speed changes by both vessels should be avoided, since that may result in a possible collision or near-miss situation.

One should note that any change in course or speed conditions by the stand-on vessel at the general collision risk region can result in a possible violation of the COLREGs, (COLREGS, 1972 Rule 8 Action to avoid collision and Rule 17 Action by stand-on vessel). Furthermore, that can confuse the give-way vessel. However, if the give-way vessel is not taking any collision avoidance actions, then the stand-on vessel should take appropriate actions to avoid a possible collision or near-miss situation. It is expected that such collision avoidance actions by the stand-on vessel should be taken in the critical collision risk region. Therefore, vessel encounter situations in the critical collision risk region should take emergency measures to avoid possible collision or near miss situations. The COLREGs do not provide adequate details on possible collision avoidance actions specially for these situations, therefore human navigator knowledge and experiences should be taken to avoid possible collision or near miss situations. Since the COLREGs require vessels to take any measures to avoid possible collision or near miss situations even when the give-way vessel is violating the 
COLREGs, the stand-on vessel should prepare for crash stop type of maneuvers to avoid possible collision or near miss situations in this region.

\section{CRITICAl COLlision Risk Situations}

\section{A. Ship Close Encounters}

Four critical risk situations, where the own vessel is having close encounter situations with the target vessel, are considered (see Figure 3, 4, 5, and 6) to identify possible regulatory failure situations. It is also assumed that the target vessel has not honored the COLREGs in these situations and that can increase the complexity in the respective ship encounter situation. Furthermore, the respective collision risk in a ship encounter situation can only be preserved by such a assumption. i.e., if both vessels take early collision avoidance actions, then the collision risk can be eliminated, quickly, therefore, none of the vessels should take any further actions. Hence, the collision avoidance framework cannot be evaluated, properly. The worst-case scenarios with higher collision risk in ship encounters are considered in this study to identify possible regulatory failure situations due to the same reasons.

Figure 3 represents a situation in the critical collision risk region, where the target vessel as the give-way vessel is heading towards a trajectory intersection point, i.e., a clear COLREGs violation with crossing from the port. Hence, the own vessel as the stand-on vessel should take necessary actions to avoid a possible collision or near miss situation. One should note that the green arrows in this figure represent the collision avoidance actions that should have been taken by the vessels in accordance with the COLREGs and the red arrows represent the collision avoidance actions that have been taken by the vessels due to the criticality of the situation i.e. can be a COLREGs violation. Therefore, the own vessel as the stand-on vessel has taken a crash stop type maneuver to make a trajectory that is parallel to the target vessel trajectory. Since no intersection between these trajectories, those actions have avoided a possible collision or near miss situation.

Figure 4 represents a situation, where the target vessel as the give-way vessel is heading towards a trajectory intersection point, a clear COLREGs violation with crossing from the port/overtaking. Hence, the own vessel as the standon vessel has taken a crash stop type maneuver to make a trajectory that is parallel to the target vessel trajectory. Since no intersection between these trajectories, those actions have avoided a possible collision or near miss situation.

Figure 5 represents a situation, where the target and own vessels are in a head-on situation with a trajectory intersection point, a clear COLREGs violation. The owe vessel has taken necessary actions, i.e. turn to the starboard, to avoid a possible collision or near-miss situation. Since no intersection between these trajectories, those actions have avoided a possible collision or near miss situation. Therefore, executing those collision avoidance actions under the proposed framework with the digital helmsman can also improve the navigation safety.
Figure 6 represents a critical collision risk situation in ship navigation. This situation has identified by executing various collision avoidance simulations under the respective collision avoidance framework. The outcome shows that this is a possible regulatory failure situation. This figure represents a situation, where the target and own vessels are heading towards the trajectory intersection point, a clear COLREGs violation with crossing from the port/head-on. The main issue in this situation is that the decision support feature had difficulties to identify this either as a crossing or head-on situation. Even the bearing between the own and target vessels may not be able to clarify this classification. Therefore, the validity and applicability of the respective stand-on and giveway vessel concepts can also be challenged. Any collision avoidance actions taken by the stand-on vessel can be either be COLREGs violations or regulatory failures.

The own vessel can take two possible crash stop type maneuvers in this situation, i.e. turn to the starboard or port. Even though the own vessel has enough navigation space, the port turn can be a violation of the COLREGs. If the target vessel decides to turn starboard as the give-way vessel, then that may result in a possible collision or near-miss situation with a trajectory intersection point. On the other hand, the starboard turn can also intercept the navigation trajectory of the target vessel. That may also result in a possible collision or near-miss situation. One should note that it would be difficult to make a crash stop maneuver from the starboard due to the angle between the respective trajectories. The outcome shows that the own vessel can stop in front of the target vessel with lost maneuverability and that can also be a possible collision or near miss situation. Therefore, executing these collision avoidance actions under the digital helmsman can challenge the navigation safety in the respective vessels in this situation. On the other hand, this situation can also happen in the general collision risk region. Therefore, the required measures to avoid such a situation should be taken.

\section{CONCLUSIONS}

Several possible regulatory failure situations are evaluated in a simulated environment under the proposed collision avoidance framework in this study. One should note that these simulations may not be able to capture all possible ship collision situations, therefore additional approaches yet to be investigated. However, this evaluation is focused in the critical collision risk region, where close ship encounter situations can be observed. One critical situation with several possible collision or near miss conditions under the digital helmsman is identified in this study. This situation is categorized as the head-on slightly to port situation and that has been further investigated to identify the respective reasons. It is noted that the critical collision avoidance actions that can be taken by the own vessel even as the stand-on vessel are limited in these situations and the outcome either be a COLREGs violation or a regulatory failure. Hence, the definitions of head-on and crossing situations and stand-on and give-way vessels are challenged in this situation even under human navigators. 


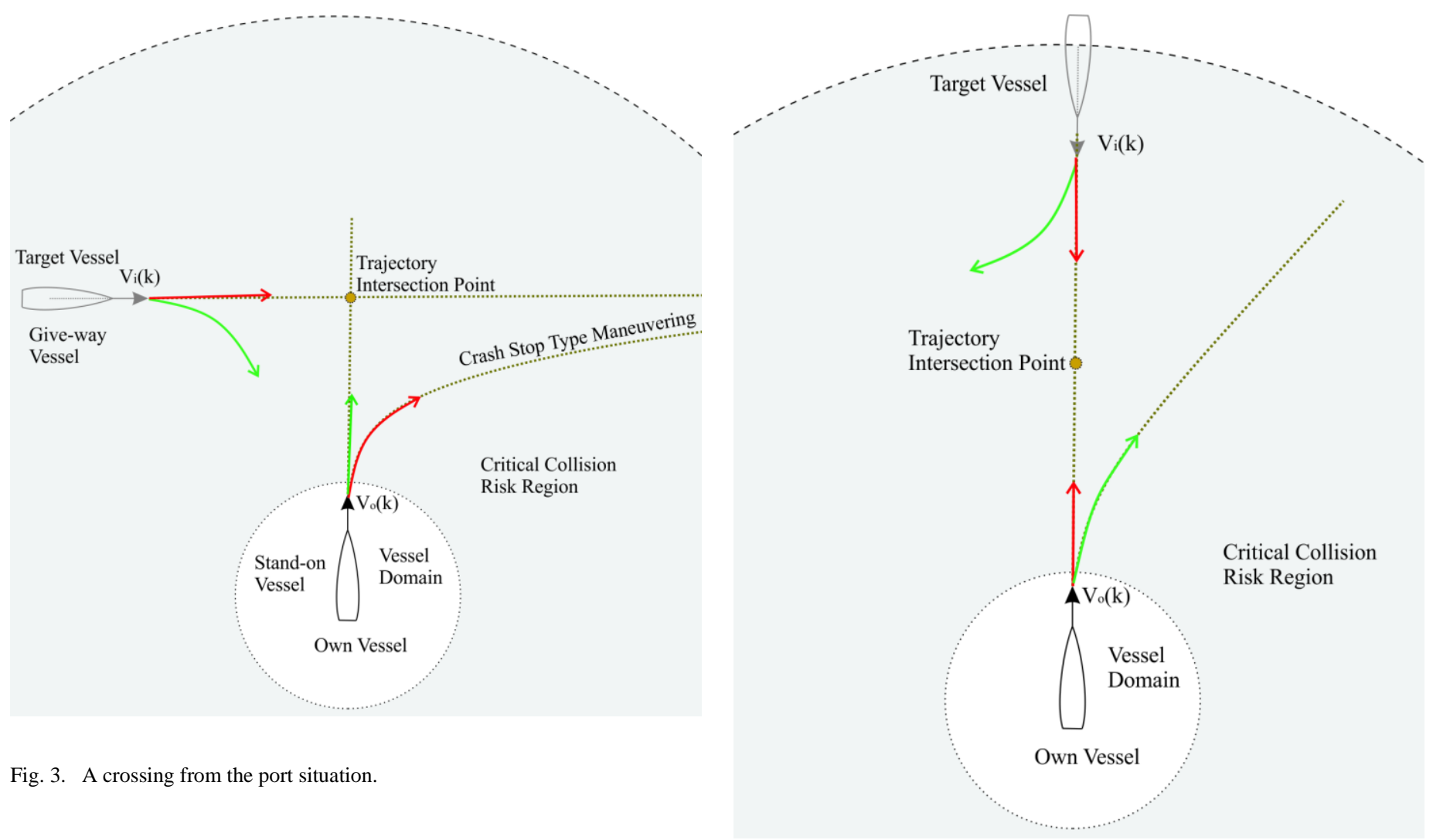

Fig. 5. A head-on situation

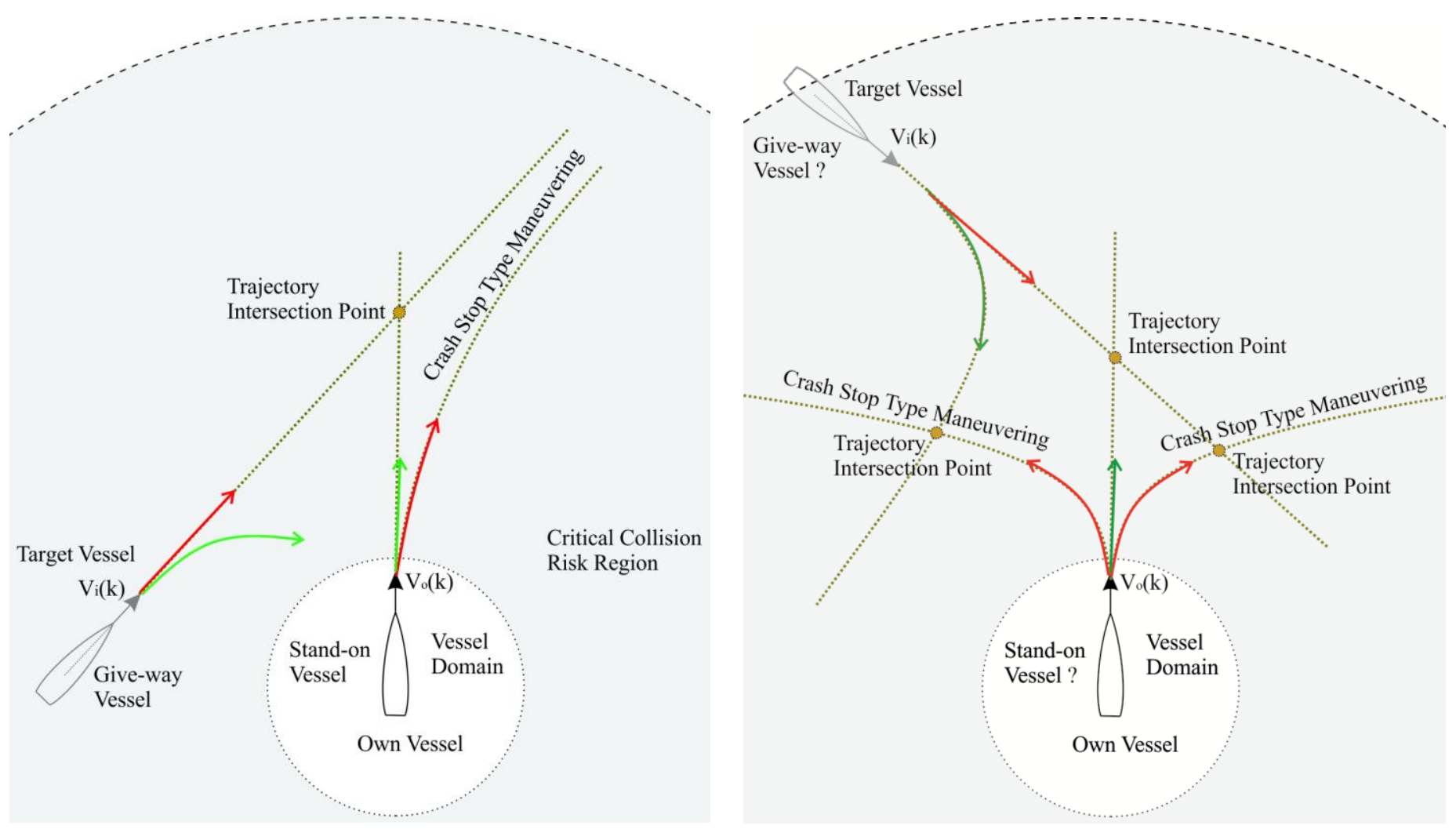

Fig. 6. A crossing from the port/head-on situation

Fig. 4. A crossing from the port/overtaking situation 
This situation can occur as an intermediate state of ship encounter situations, i.e. in both the general collision risk region and critical collision risk region. Such situations cannot be ignored as an improbable or isolated situation due to these reasons. Furthermore, the complexity in this critical collision situation can be increased in several directions and that are discussed in the following section.

These close ship encounters are further simplified by assuming that the vessels are moving in straight line trajectories and their course-speed vectors and headings are in the same direction. These assumptions may not hold in realistic ocean-going situations, therefore the following outcomes are expected:

- If the course-speed and heading vectors in these vessels are having significant differences; therefore these vessels can be moving in parabolic type maneuvers; then that can introduce additional complexities into the collision risk estimation.

- If this situation is associated with more than two vessels, various collision avoidance decisions can overlap and even cancel each other in some situations.

- If any constrains in the vessel maneuverability can introduce additional difficulties in executing collision avoidance actions.

These outcomes can further degrade the collision avoidance actions that are taken by humans in critical collision situations. Furthermore, the respective collision avoidance actions, by the digital helmsman can also be challenged in such a situation, since the respective decisions are based on a set of navigation rules and regulations, i.e. the COLREGs. Therefore, adequate solutions to overcome such critical collision situations within the COLREGs should be investigated to make autonomous shipping a reality.

\section{ACKNOWLEDGMENT}

This work has been conducted under an internal funded project of UiT The Arctic University of Norway - that supports towards autonomous ship navigation in the arctic region. This work is also supported by the MARKOM2020 project, a development project for maritime competence established by the Norwegian Ministry of Education and Research in cooperation with the Norwegian Ministry of Trade and Industry

\section{REFERENCES}

[1] O. J. Rodseth, L. P. Perera, and B. Mo,"Big data in shipping Challenges and opportunities," In Proceedings of the 15th International Conference on Computer Applications and Information Technology in the Maritime Industries (COMPIT 2016), Lecce, Italy, May, 2016, pp. 361-373.

[2] L.P. Perera and B. Murray, "Situation Awareness of Autonomous Ship Navigation in a Mixed Environment under Advanced Ship Predictor," In Proceedings of the 38th International Confference on Ocean, $\mathrm{O}$ shore and Arctic Engineering (OMAE 2019), Glasgow, Scotland, UK, June, 2019 (OMAE2019-95571).

[3] L. P. Perera,"Autonomous Ship Navigation under Deep Learning and the challenges in COLREGs," In Proceedings of the 37th International Conference on Ocean, Offshore and Arctic Engineering (OMAE 2018), Madrid, Spain, June, 2018 (OMAE2018-77672).
[4] A. Fridman, et al., MIT Autonomous Vehicle Technology Study: LargeScale Deep Learning Based Analysis of Driver Behavior and Interaction with Automation lessArXiv2017.

[5] L.P. Perera, J.P. Carvalho and C. Guedes Soares, "Intelligent ocean navigation \& Fuzzy-Bayesian decision-action formulation," IEEE Journal of Oceanic Engineering, vol 37, no 2, 2012, pp 204-219.

[6] M. L. Smith, "Sensors, appliance control and fuzzy logic," IEEE Transactions on Industrial Applications, vol. 30, no. 2, pp. 305-310, 1994.

[7] T. Ozen, J. M. Garibaldi, and S. Musikasuwan, "Modeling the variation in human decision making," in IEEE Annual Meeting of the Fuzzy Information Processing - NAFIPS 04, vol. 2, June 2004, pp. 617-622.

[8] L.P. Perera, V. Ferrari, F.P. Santos, M.A. Hinostroza, and C. Guedes Soares, "Experimental Evaluations on Ship Autonomous Navigation \& Collision Avoidance by Intelligent Guidance," IEEE Journal of Oceanic Engineering, vol. 40, no. 2, 2015, pp 374-387.

[9] IMO, COLREGS - International Regulations for Preventing Collisions at Sea, 1972.

[10] L.P. Perera, J. P. Carvalho and C. Guedes Soares, "Fuzzy-logic based decision making system for collision avoidance of ocean navigation under critical collision conditions," Journal of Marine Science and Technology, vol 16, 2011, pp 84-99.

[11] L.P. Perera, and C. Guedes Soares, "Collision Risk Detection and Quantification in Ship Navigation with Integrated Bridge Systems," Journal of Ocean Engineering, vol. 109, 2015, pp. 344-354.

[12] B. Murray and L.P. Perera, "AIS-based Probabilistic Vessel Trajectory Prediction for Collision Avoidance in Future Vessels," In Proceedings of the 38th International Conference on Ocean, Offshore and Arctic Engineering (OMAE 2019), Glasgow, Scotland, UK, June, 2019 (OMAE2019-95963).

[13] L.P. Perera, J.P. Carvalho and C. Guedes Soares, "Solutions to the Failures and Limitations of Mamdani Fuzzy Inference in Ship Navigation," IEEE Transactions on Vehicular Technology, vol. 63, no. 4, 2014, pp 1539-1554.

[14] L.P. Perera and B. Murray, "Situation Awareness of Autonomous Ship Navigation in a Mixed Environment under Advanced Ship Predictor," In Proceedings of the 38th International Conference on Ocean, O shore and Arctic Engineering (OMAE 2019), Glasgow, Scotland, UK, June, 2019 (OMAE2019-95571). 